\title{
La « spécificité » de la biologie : une comparaison avec la physique
}

\section{Claudio Longo et Enrico Giordano}

\section{(2) OpenEdition \\ 1 Journals}

Édition électronique

URL : http://journals.openedition.org/trema/1976

DOI : 10.4000/trema.1976

ISSN : 2107-0997

Éditeur

Faculté d'Éducation de l'université de Montpellier

\section{Édition imprimée}

Date de publication : 1 septembre 1996

Pagination : 16-22

ISSN : 1167-315X

\section{Référence électronique}

Claudio Longo et Enrico Giordano, « La «spécificité » de la biologie : une comparaison avec la

physique ", Tréma [En ligne], 9-10 | 1996, mis en ligne le 11 septembre 2013, consulté le 19 avril 2019. URL : http://journals.openedition.org/trema/1976 ; DOI : 10.4000/trema.1976

Ce document a été généré automatiquement le 19 avril 2019

Trema 


\title{
La « spécificité » de la biologie : une comparaison avec la physique
}

\author{
Claudio Longo et Enrico Giordano
}

\section{Introduction}

1 Notre groupe de travail est constitué d'un biologiste (C.L.), d'un physicien (E.G.), d'un didacticien (Graziano Cavallini) et d'une dizaine d'enseignants d'école secondaire inférieure («Scuola Media»: 11-13 ans). Nous cherchons à établir des curricula de sciences s'étendant sur les trois années d'école secondaire inférieure. Nous expérimentons ces curricula dans les classes et nous étudions l'évolution intellectuelle des élèves pendant ces trois ans. Nos différentes compétences professionnelles nous permettent d'obtenir un curriculum assez bien équilibré entre la biologie et la physique (Giordano et Longo, 1990).

2 Pendant les longues années de notre collaboration, nous avons eu de nombreuses occasions de discuter à propos des caractères spécifiques de la physique et de la biologie. La présence d'un didacticien parmi nous catalysait, peut-être, ces discussions «philosophiques ».

3 On pourrait considérer que ces discussions ne constituent qu'un sous-produit de notre activité par rapport aux "produits primaires » (activités de classe, observations sur les élèves, etc.). Nous pensons au contraire que ces discussions sont un produit très important de notre activité parce qu'elles peuvent avoir un effet immédiat sur l'enseignement. Si l'enseignant comprend mieux le caractère spécifique de la biologie, il pourra mieux l'enseigner parce qu'il pourra mieux distinguer entre l'essentiel et l'accessoire. Mais ce caractère spécifique ne prend de sens que par rapport aux autres disciplines scientifiques. En conséquence, on ne peut pas bien comprendre le caractère spécifique de la biologie si on ne comprend pas le caractère spécifique des autres disciplines, par exemple de la physique. 
Cette comparaison est particulièrement importante aux premiers niveaux de la scolarité où le même enseignant intervient dans des disciplines scientifiques différentes. Par exemple, à l'école secondaire inférieure, le même professeur enseigne mathématiques et sciences tandis qu'à l'école secondaire supérieure nous disposons de deux enseignants différents pour biologie, chimie, sciences de la terre d'une part, mathématique et physique de l'autre.

Dans les pages suivantes nous donnerons quelques exemples des «résultats » de nos discussions.

\section{Biologie et physique : deux pôles opposés ?}

$6 \quad$ Les différences entre biologie et physique sont vraiment profondes. On peut imaginer que les autres disciplines scientifiques ont une position intermédiaire entre les deux: la chimie étant un peu plus proche de la physique et les sciences de la terre un peu plus proches de la biologie.

7 La physique est universellement considérée comme plus difficile que la biologie. La raison principale est que notre langage naturel n'est pas mathématique. Mais d'un autre point de vue, la physique est peut-être moins complexe : la biologie est beaucoup plus riche et diversifiée et les facultés mentales nécessaires pour la maîtriser sont plus diverses.

Le monde de la physique est fait d'un nombre assez petit d'«ingrédients ». En simplifiant beaucoup, ces «ingrédients » pourraient être : recherche de variables significatives aux objectifs du problème qu'on veut étudier, mesure de variables, recherche de relations mathématiques entre variables mesurées, modélisation qui peut arriver, dans certains cas extrêmes, au niveau d'une théorie englobant tout l'univers.

9 On peut totalement appliquer à la biologie le point de vue de la physique, mais en procédant ainsi, on l'appauvrit terriblement. Bien sûr, on retrouve en biologie la recherche et la mesure de variables, la formalisation mathématique, la modélisation, mais on y trouve aussi d'autres éléments qu'on ne peut absolument ramener aux précédents (Mayr, 1982) et que nous décrirons ensuite.

10 Malheureusement, beaucoup de biologistes éprouvent des complexes d'infériorité envers la physique (et la chimie aussi). Ils estiment que la biologie doit être mathématisée et formalisée autant que la physique tandis que tous les autres aspects (par exemple la description) sont à mépriser (Hull, 1974). Malheureusement, ces biologistes sont souvent des professeurs d'université et leur attitude finit ainsi par influencer le contenu des enseignements de biologie et la formation des enseignants.

11 La biologie est biologie parce qu'on ne peut pas l'assimiler totalement à la physique : autrement ce serait seulement de la physique appliquée. Les grandes questions de la biologie ne peuvent pas trouver une réponse seulement clans le domaine de la physique (ni dans celui de la chimie non plus).

\section{L'élément descriptif}

12 Un de ces caractères particuliers de la biologie est la description. En biologie on décrit à tous les niveaux: les caractères typiques de l'espèce Fragaria vesca ou Bufo viridis, la séquence de bases d'un gène mais aussi la séquence des événements qui se succèdent 
pendant la digestion des aliments. La physique, au contraire, est très pauvre de ce point de vue. On ne décrit pas un électron ou un neutron : ils n'ont ni forme ni couleur et leur comportement est mieux décrit par une équation que par des mots. Bien sûr, en optique on décrit une lentille biconvexe, mais c'est une description pauvre où tout détail est fourni dans un but précis, tandis qu'en biologie on décrit souvent objets et événements seulement " parce qu'ils sont là » sans arrière-pensées.

13 Comme nous l'avons dit précédemment, beaucoup de chercheurs et aussi d'enseignants de biologie sont embarrassés par cet aspect de leur discipline qui ne correspond pas à leur idée de science " pure et dure » et trouvant cela humiliant, ils cherchent à le dissimuler. «Le biologiste n'est pas un chasseur de papillons ou un ramasseur de fleurs » : combien de fois avons-nous entendu cette phrase! Souvent les enseignants pensent que la physique (comme les mathématiques) implique les plus hautes facultés de l'esprit (raisonnement abstrait, etc.) tandis que la biologie exige surtout la mémoire (en Italie, on dit avec mépris « apprendre par cœur l'annuaire téléphonique »).

Un escamotage souvent utilisé pour éviter la description systématique des reptiles, des amphibiens, des gymnospermes... consiste à se rabattre sur l'argument de la « diversité de la vie ». Ainsi on peut réduire la diversité à des principes généraux de classification ou encore à la question : comment cette diversité s'est-elle instaurée ? Dans ce cas, il suffit de fournir quelque exemple et la honteuse complexité devient profondeur aristocratique.

Mais cela est inutile car si on peut cacher le problème, on ne peut pas l'abolir. En biologie il faut souvent décrire sans pouvoir s'appuyer sur des hypothèses pour interpréter tout de suite ce qu'on observe - et surtout il ne faut pas en avoir honte. Généralisation et modélisation viendront après.

Crapaud vert ou « Bufo viridis ».

En France, on ne le trouve qu'en Savoie et en Corse.

ROMER A., L'Évolution Animale - Tome II, Lausanne-Suisse, Éditions Rencontre, 1970.

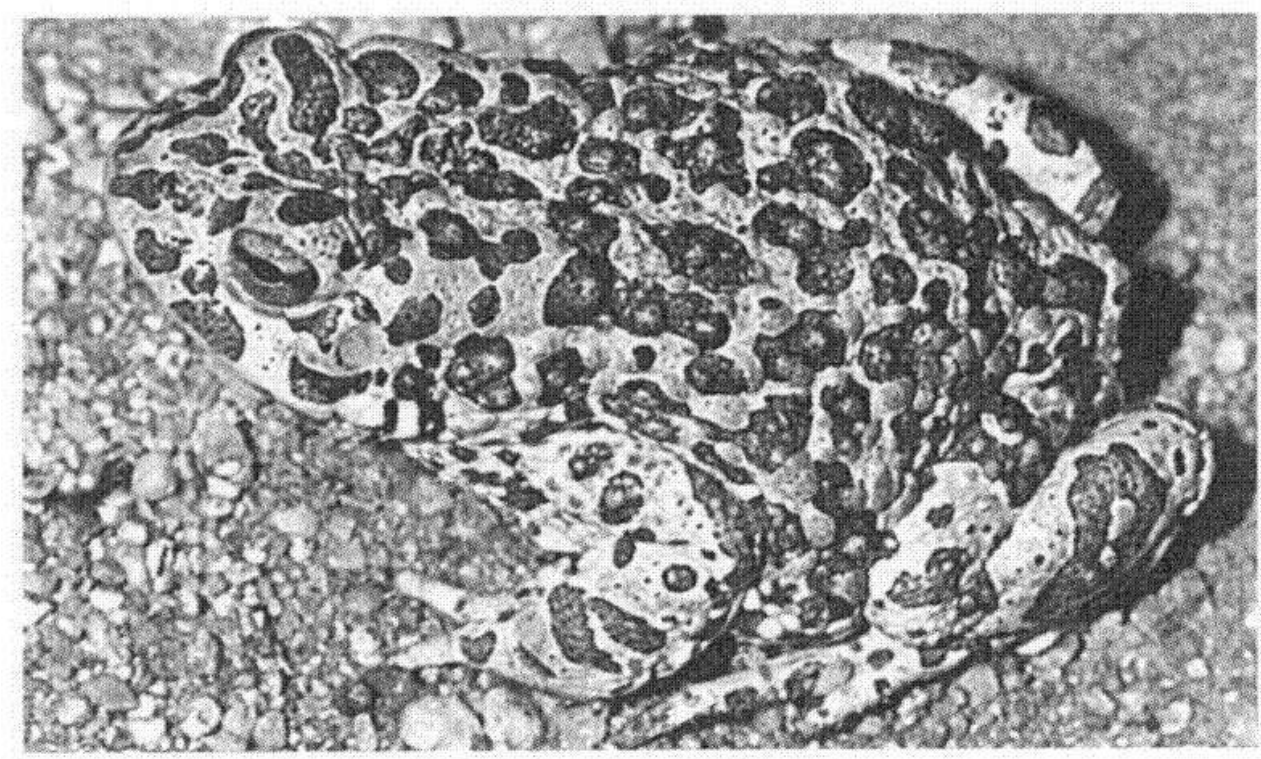




\section{L'aspect historique}

16 Cet aspect a une importance fort différente en biologie et en physique. En physique il concerne seulement l'histoire de la discipline, en biologie il concerne l'histoire de la discipline et l'histoire de la vie en même temps, et dans certains cas (Darwin, etc.), les deux histoires sont inséparablement liées. Le point de vue historique est peut-être un luxe dans l'enseignement de la physique, mais pour l'enseignement de le biologie il est d'une nécessité absolue. En ignorant Galileo, Newton, Einstein on peut encore enseigner une physique très grossière, mais cohérente - mais une biologie sans Darwin et sans évolution est tout à fait impossible! En biologie on retrouve l'évolution partout, même là où on n'imaginerait jamais la trouver- par exemple quand on parle de l'apparition de bactéries pathogènes résistantes aux antibiotiques.

\section{Le rôle de la technologie}

Biologie et physique sont tout à fait opposées sous cet aspect. La physique est nécessaire pour comprendre la technologie, mais à son tour la technologie est utile, pour ne pas dire indispensable pour apprendre la biologie. La première affirmation est évidente, la seconde exige, peut-être, une explication.

18 Un organisme vivant ressemble à une machine très complexe. Cette idée est ancienne (Descartes, etc.), mais elle est encore valable. Un grand nombre de propriétés des organismes peuvent être très bien expliquées par la comparaison avec une machine.

19 Cependant, la plus grande utilité de la technologie n'est pas de permettre de comprendre les menus détails du fonctionnement des organismes, mais de saisir quelques grands principes, par exemple les idées de régulation automatique, de rétroaction, etc.

20 Voici un exemple d'application de la technologie à l'enseignement biologique. Toutes les régulations dans les organismes vivants sont automatisées: il ne pourrait en être autrement parce qu'il n'y a pas un «Créateur » qui peut intervenir de l'extérieur sur les mécanismes de régulation. On peut enseigner ce concept en comparant un appareil à régulation manuelle avec un autre à régulation automatique. L'exemple le plus simple pourrait être celui du climatiseur automatisé d'une voiture de luxe et du conditionneur d'air manuel d'une voiture moins chère.

21 La technologie nous ramène à une différence des plus importantes entre biologie et physique : l'idée de finalité, de fonction. Cette idée est tout à fait étrangère à la physique (quelle est l'utilité d'un pion? - une question absurde) tandis qu'elle est véritablement au centre de la biologie (Monod, 1970). Et peu importe qu'en biologie, à la différence de la technologie, il ne s'agisse que d'une finalité seulement apparente (l'évolution est aveugle !). Presque tous les détails de la structure d'un organisme peuvent être expliqués par l'idée de finalité et de fonction- tout se passe exactement comme s'il y avait une finalité fixée d'avance.

\section{Laisser de la place aux émotions !}

22 L'impact émotionnel de la biologie est énorme. Aucune discipline scientifique ne lui est comparable. Sexe, mort, succession de générations, manger et être mangé, compétition, 
symbiose... Imaginez en comparaison : accélération, champ électrique, proton, indice de réfraction - la biologie est clairement autre chose!

(Il n'est pas vrai que la physique soit complètement dépourvue d'éléments émotionnels pensez par exemple à l'entropie, mais il faut une mentalité assez abstraite pour les ressentir et les apprécier).

Le problème pour un enseignant est : que faire des émotions ? Les ignorer, les combattre, les exploiter ? C'est probablement la première option qui est la plus commune, mais nous suggérons fortement de choisir la troisième. Les aspects émotionnels peuvent valoriser la discipline parce que les élèves se sentent impliqués. "Implication » représente quelque chose de plus fort qu' "intérêt»: on pourrait dire qu' "implication» présente une température psychologique plus élevée.

L'enseignant qui doit aborder l'écologie pourrait donner de la place au sens de la beauté de la nature qui s'est manifesté au cours des siècles à travers la poésie, la peinture, la musique... Plaisir pour la beauté de la nature, chagrin pour la nature défigurée peuvent parfaitement coexister avec l'explication plus scientifique possible de l'eutrophisation ou de la destruction de la couche d'ozone : il n'y a pas d'interférence destructive entre les deux points de vue.

Au contraire, à notre avis la science trop froide est un danger. On pourrait faire là une analogie avec la chimie : à basse température les molécules ne réagissent pas ensemble, ne se transforment pas. De la même façon, une température émotionnelle trop basse empêche la formation de liens entre connaissances différentes : les connaissances restent isolées au lieu de réagir ensemble.

Conclusion: l'implication émotionnelle typique de la biologie n'est pas un ennemi à combattre : au contraire, c'est un avantage à exploiter !

\section{Physique et biologie à l'université}

Les étudiants de biologie à l'université apprennent la physique comme matière auxiliaire pouvant fournir des connaissances utiles pour certains domaines de la biologie, par exemple la physiologie. Nous croyons que considérer la physique seulement comme matière auxiliaire sans entrer dans son univers culturel en fait une bien pauvre chose. Les étudiants peuvent mieux apprécier les particularités de la biologie s'ils ont un terme de comparaison tout à fait différent. Physique et biologie ont toutes les deux à gagner si les enseignants mettent en évidence les caractères spécifiques des deux disciplines. Si l'on suit cette voie, la science devient culture.

Cette comparaison est plus facile à l'école élémentaire ou à l'école secondaire inférieure où le même professeur enseigne les deux matières, mais elle est aussi possible à l'école secondaire supérieure ou à l'université (biologie, sciences naturelles, médecine, etc.) si les enseignants de physique et de biologie sont disposés à collaborer.

\section{Conclusion}

Dans l'enseignement de la biologie il faudrait tenir compte de trois aspects différents : a) les aspects typiques de toute méthode scientifique (hypothèse, planification d'expériences, etc) qui sont à peu près les mêmes pour toutes les disciplines; b) les 
aspects quantitatifs, formels qui ressemblent à ceux de la physique ; c) les aspects qui rendent la biologie différente des autres disciplines: description, finalisme, implication émotionnelle...

\section{BIBLIOGRAPHIE}

GIORDANO E. \& LONGO C., « Le mouvement du point de vue de la zoologie et de la physique dans le collège (11-14 ans) », « Est-il possible de mélanger la fantaisie avec la rigueur logique ?», in : Giordan. A., Martinand J.-L. \& Souchon C, Sciences, Techniques et Imaginaire, Actes des XII Journées Internationales sur la communication, l'éducation et la culture scientifiques et industrielle, Chamonix, 1990, p. 467-469.

HULL D, Philosophy of biological science, Englewood Cliffs, New Jersey, USA, Prentice Hall, 1974.

MAYR E., The growth of biological thought. Diversity, evolution and inheritance. Cambridge, Massachusetts. USA, Belknap Press, 1982.

MONOD J., Le hasard et la nécessité, Paris, Éditions du Seuil, 1970.

\section{RÉSUMÉS}

Les discussions et les débats portant sur les caractères particuliers de la biologie comparée à la physique permettent d'orienter l'enseignement. De nombreux caractères rendent la biologie profondément différente de la physique : a) l'élément purement descriptif ; b) le recours à la technologie pour exprimer des concepts importants ; c) l'importance essentielle de l'élément historique (histoire de la discipline, histoire de la vie) ; d) l'extraordinaire impact émotionnel.

Discussions and debates bearing upon biology's specific nature as compared to physic's make it possible to give a specific slant to its teaching. A number of characteristics make biology profoundly different, from physics: a) the utterly descriptive, factor; b) its recourse to technology to express important concepts; e) the essential importance of the historical element (history of the discipline, history of life); d) its extraordinary emotional impact.

\section{INDEX}

Mots-clés : biologie, description, didactique, émotion, physique

Keywords : biology, didactic, physic

\section{AUTEURS}

\section{CLAUDIO LONGO}

Dipartemento di Biologia, via Celoria, 26, Milano, Italia 


\section{ENRICO GIORDANO}

Instituto di Fisica Sperimentale e Applicata, via Celoria, 20, Milano, Itala 